\title{
Strong variations of gamma-ray and atmospheric electric field during various meteorological conditions by observations in Yakutsk and Tiksi
}

\author{
Anatoliy Toropov ${ }^{1,1}$, Sergey Starodubtzev ${ }^{1}$, Vladimir Kozlov ${ }^{1,2}$ \\ ${ }^{1}$ Yu.G. Shafer Institute of Cosmophysical Research and Aeronomy SB RAS, 677980 Lenin Ave, 31, \\ Yakutsk, Republic of Sakha (Yakutia), Russian Federation \\ ${ }^{2}$ Physical Technical Institute of the North-Eastern Federal University, 677000 Belinsky St., 58, \\ Yakutsk, Republic of Sakha (Yakutia), Russian Federation
}

\begin{abstract}
The results of a study strong increase in gamma-ray background in the surface layer of the atmosphere during precipitation and near thunderstorms in Yakutsk (based on the Cosmic Ray Spectrometer of the Institute of Cosmic Rays of the Siberian Branch of the Russian Academy of Sciences) and Tiksi (Polar Geospace Observatory of the IKFIA SB RAS) are present in this paper. Scintillation detectors based on $\mathrm{NaI}(\mathrm{Tl})$ crystals with a size of $63 \mathrm{~mm} \times 63 \mathrm{~mm}$ are used to register gammaray . The range of measured energies is $20-1900 \mathrm{keV}$. Detectors are closed from the sides and from below with lead $(5 \mathrm{~cm})$ and placed in thermoboxes having a glass window. Both of the detectors are located on the roof of buildings in Yakutsk and Tiksi. Also, synchronous registration of variations of the atmospheric electric field, (electrostatic fluxmeters Boltek EFM-100) and main meteorological parameters of the atmosphere (ultrasonic weather station AMK-04). An explicit relationship was found between the increase in the background gamma-ray and heavy rainfall. The energy spectra of the gamma background are obtained under "good" weather conditions and during heavy precipitation and near thunderstorms. The carried out researches allow to consider that the increase of the gamma background is connected, first of all, with the emission of radon and its daughter products from the ground and, to a lesser extent, with bremsstrahlung of charged particles in strong electric fields of a thunderstorm atmosphere.
\end{abstract}

\section{Introduction}

Numerous studies of recent decades describe in detail the results of studies of variations in the surface gamma background. Strong variations of the gamma background during intense precipitation [1] and in a thunderstorm atmosphere [2] have been repeatedly noted. There is still no single point of view on the mechanism of such variations of the gamma background. The most popular opinion is that variations in the gamma background during

1 Corresponding author: toropov@ikfia.ysn.ru 
heavy precipitation are due to radon emission from the soil and precipitation of radon decay products $(210 \mathrm{Po}, 210 \mathrm{~Pb}, 214 \mathrm{~Pb}, 214 \mathrm{Bi})$ on aerosols and water droplets [3]. According to another theory [4], the increase in gamma background is due to the bremsstrahlung of cosmic ray muons in strong electric fields of a thunderstorm atmosphere. Another theory [5], suggests the existence of powerful gamma-ray flashes during lightning discharges (TGF). The cosmogenic nature of increases in the surface gamma background cannot be excluded $[6,7,8]$. The cosmogenic isotope of beryllium $(\mathrm{Be} 7)$ is formed in the upper layers of the atmosphere under the influence of cosmic rays and slowly drifts (is deposited) on the surface of the earth. Meteorological phenomena such as thunderstorms and heavy precipitation can theoretically increase the influx of cosmogenic beryllium from the upper atmosphere to the surface of the earth, thereby increasing the intensity of the surface gamma background.

These prerequisites determined the need for a series of field observations in Yakutsk and Tiksi related to the study of variations of natural gamma radiation during precipitation, near thunderstorms, snowstorms and to establish the role of variations in the atmospheric electric field, meteorological conditions of the atmosphere, the contribution of short-lived uranium radionuclides contained in aerosols in the lower atmosphere, to increase the surface gamma background. In addition, these observations are a continuation of the experimental work [9] carried out at the Institute of Cosmophysical research and aeronomy of the Siberian Branch of the Russian Academy of Sciences on the study of generation of penetrating radiation and energetic particles in the atmosphere during near thunderstorms.

\section{Equipment}

Registration of gamma background in Yakutsk and Tiksi is carried out since 2016. Both gamma detectors are absolutely identical, both in terms of their characteristics and structurally, and are made on the basis of a scintillation spectrometric sodium iodine crystal $\mathrm{NaI}(\mathrm{Tl})$ with a cylindrical shape measuring 60x60 mm (diameter and height). The scintillator, photomultiplier tube, preamplifier and high voltage converter are integrated in one case.

Gamma detectors allow you to record gamma radiation in the energy range of $20-1800$ $\mathrm{keV}$. Before setting on registration and periodically, during the year, the detectors are calibrated with three weakly active isotopes: Americium 241 (Am 241. $60 \mathrm{keV}$ line), radium 226 isotope (Ra226, daughter decay products lines: $82.1 \mathrm{keV} ; 188 \mathrm{keV} ; 241,98$ $\mathrm{keV}$; $295.21 \mathrm{keV} ; 351.92 \mathrm{keV}$; $609.31 \mathrm{keV}$ ), Cobalt 60 (Co60, two lines: $1.173 \mathrm{MeV}$; 1.333 $\mathrm{MeV})$, which allows you to test its performance in the entire range of investigated energies.

Detectors in Yakutsk and Tiksi are placed in individual thermal boxes and installed on the roof of the building of the cosmic ray spectrograph IKFIA in Yakutsk $\left(61^{\circ} 59\right.$ 'N, $129^{\circ}$ $\left.41^{\prime} \mathrm{E}\right)$ and on the roof of the building of the neutron monitor of the Polar Geocosmophysics Observatory of ShIKRA in Tiksi $\left(71^{\circ} 35^{\prime} \mathrm{N}, 128^{\circ} 46^{\prime} \mathrm{E}\right)$. Figure 1 shows the device and appearance of the gamma detector. Thermoboxes have built-in heaters with a thermostat and are designed to operate at low (up to -50 degrees Celsius) temperatures. The body of the thermobox is made of steel. On the door of the thermobox there is a glass window opposite which a gamma detector is fixed. 


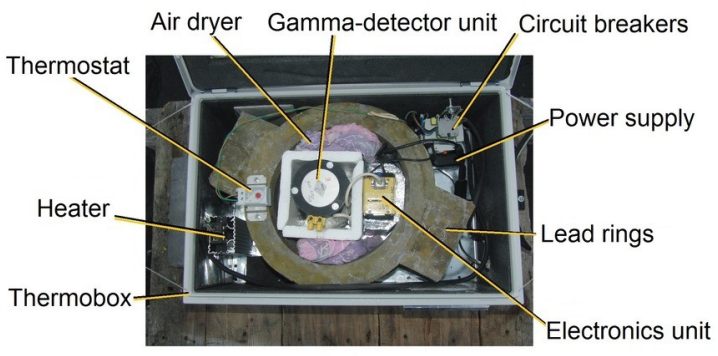

(a)

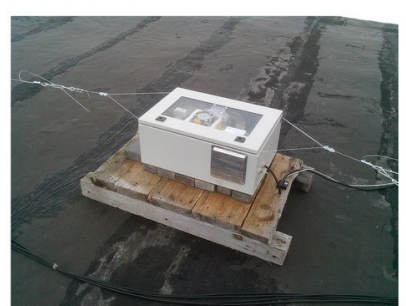

(b)

Fig. 1. Internal structure (a) and appearance (b) of a gamma detector.

To reduce the influence of background gamma-radiation from the soil, the detector is installed inside lead rings with a wall thickness of $50 \mathrm{~mm}$, and a pad of 12 lead bricks 50 $\mathrm{mm}$ thick is made under the base of the thermobox. Thus, the detector is most sensitive to gamma radiation coming in from the direction of the zenith. The maximum aperture (taking into account the position of the $\mathrm{NaI}(\mathrm{Tl})$ scintillator in the rings) is approximately $60-65$ degrees from the vertical. In addition, due to the large mass $(150 \mathrm{~kg})$, the lead inside the thermobox performs the function of additional thermal stabilization. The total weight of the detector (with lead protection) is approximately $300 \mathrm{~kg}$.

Data collection and storage from detectors in Yakutsk and Tiksi is carried out using the Arduino MEGA 2560 card. Data is transferred to a computer via a USB port. In this article uses data with a minute resolution.

In addition, at the Yakutsk station, the analog signal from the gamma detector is recorded using the high-speed E-20-10 ADC (manufactured by L-Card), which allows continuous data collection with a signal digitizing frequency of up to $10 \mathrm{MHz}$ at $14 \mathrm{bits}$. Using special software written in the Lab-View language, separate pulses are allocated, their amplitude is determined and the real-time gamma-ray spectrum is built. The accumulation time of one spectrum is 5 minutes. Later, in data processing, it is possible to obtain spectra with an accumulation time of arbitrary duration.

In the immediate vicinity of the gamma-ray detectors the electric field sensor Boltek EFM-100 (an electrostatic field-mill) was installed to register the atmosphere electric field variations. The electrostatic field-mill was calibrated in an artificial electric field and has a measurement range of $+/-20 \mathrm{kV} / \mathrm{m}$. Data with a resolution of one second is used in the article.

The basic meteorological parameters of the atmosphere are recorded using an ultrasonic weather station AMK-04, produced by IMKES, Tomsk.

To assess the relationship between the variations in surface gamma radiation and the muon intensity of the secondary component of cosmic rays, as predicted in [4], the data of two muon telescopes installed on the spectrograph of cosmic rays in Yakutsk were also used. The first muon telescope was made on SGM-13 gas-discharge counters, the second on the basis of SC-301 flat scintillation detectors with an effective area of $8 \mathrm{~m} 2$. Both muon telescopes allow you to record muons with energies above $2 \mathrm{GeV}$ and the direction of their arrival. The article uses data with hourly resolution.

\section{Experimental data and results}

Figure 2 shows a graph of the event in a gamma background during a near thunderstorm on August 3, 2017. at the observation station Yakutsk. At the stage of maximum development of thunderstorms, there are strong variations in the intensity of the 
atmospheric electric field (upper panel) and a significant increase in the count rate in the gamma detector (upper panel) from 1800 pulses / min to 3000 pulses / min, which is approximately $66 \%$ of the background level. The sharp increase in the intensity of the gamma background starts at 12: 20UT and almost linearly decreases to 14: 30UT. UT there is a slight kink, and then the level of gamma background returns to its original values of about 16-17 UT.

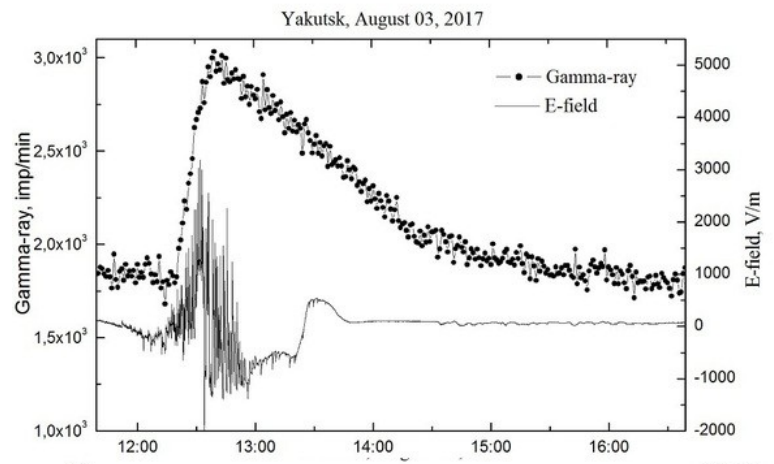

(a)

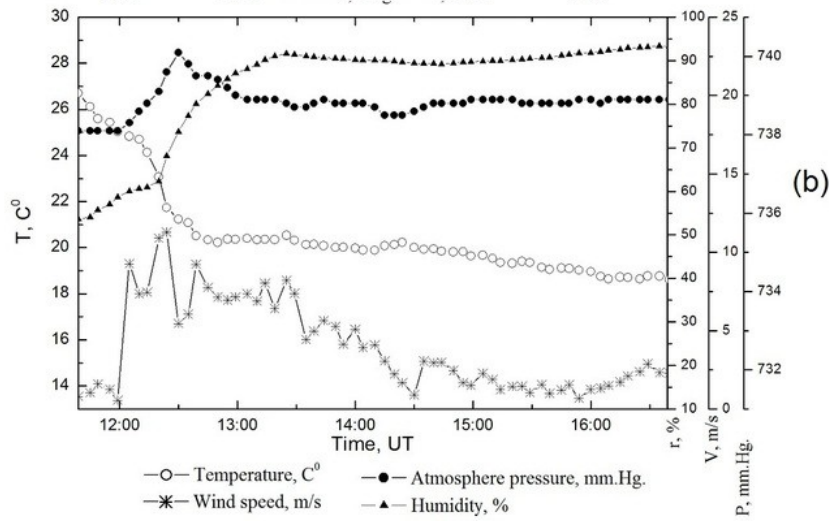

Fig. 2. Strong variations in gamma radiation (a), atmospheric electric field (a) and basic meteorological parameters of the atmosphere (b) during a thunderstorm on August 3, 2017 in Yakutsk.

At the same time, significant variations are observed in the basic meteorological parameters of the atmosphere (lower panel) characteristic of thunderstorms. Meteorological parameters such as humidity and atmospheric pressure tend to increase. Moreover, the atmospheric pressure has a maximum in the form of a peak in time (12:30 UT) coinciding with the maximum of the intensity of gamma radiation. On the contrary, the air temperature decreases from 26 to 19 degrees. The wind speed greatly increase at the time of the beginning of a thunderstorm from $2 \mathrm{~m} / \mathrm{s}$ to $10-11 \mathrm{~m} / \mathrm{s}$ and gradually decreases to prestorm values at about $16 \mathrm{UT}$.

Special attention should be paid to the fact that a high intensity of gamma radiation is also observed after a thunderstorm (starting at 13:40 UT) in the absence of strong electric fields. The absence of a close connection between variations in the surface atmospheric electric field and variations in gamma radiation suggests, probably, that the strong electric fields of a thunderstorm atmosphere are not the main cause of a strong increase in the surface gamma radiation. At the same time, a clear link can be traced between gamma radiation and wind speed, and, to a lesser extent, with the value of atmospheric pressure.

It can be assumed that the increase in wind speed intensifies the processes of mixing of the surface layer of air containing radon and its daughter decay products, which under the 
action of precipitation are forced out of the pores of the soil to the surface. In turn, an increase in atmospheric pressure increases their concentration per unit volume. In this case, the characteristic lines of gamma radiation corresponding to the daughter products of the decay of radon should appear in the spectra of gamma - radiation obtained during this event.

Figure 3 shows the gamma spectra during the event of August 3, 2017. Two spectra (a) were obtained with an exposure of 3 hours before a thunderstorm and 3 hours during a thunderstorm. (b) shows the difference between these two spectra obtained by subtracting the spectrum in fair weather from the spectrum obtained in a thunderstorm. Several peaks appeared quite well which can be identified as the daughter decay products of radon $\mathrm{Pb}-214$ and Bbi-214 as well as a peak not related to them with an energy of $1008 \mathrm{keV}$, the closest isotope of it is Al-27 (1014 keV).
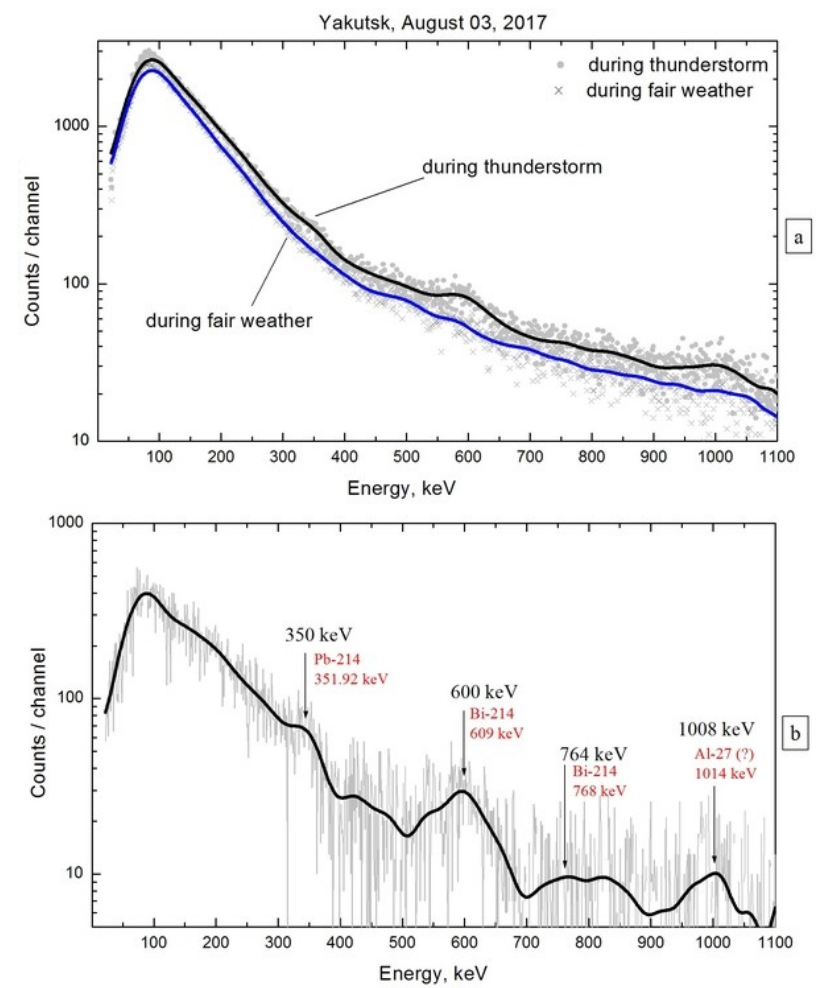

Fig. 3. The gamma spectra of radiation before a thunderstorm (blue line (a)) and after a thunderstorm (black line (a)) were obtained with an exposure of 3 hours (a) on August 3, 2017 in Yakutsk and the difference of the two spectra (b). One can see the manifestation of some characteristic lines of the daughter products of the decay of radon.

According to observations in Tiksi, from 2016, repeated increases in the intensity of surface gamma radiation during precipitation and heavy snowstorms were also observed. The most interesting events are associated with strong snowstorms, since the frozen ground should block the emission of radon and its daughter products to the surface layer of the atmosphere. In addition, variations in the intensity of the atmospheric electric field in snowstorms in Tiksi reach extreme values. 
Figure 4 shows an event during a heavy snowstorm in Tiksi on January 13,14,15, 2017. A trend is observed here that is similar to that observed in Yakutsk.

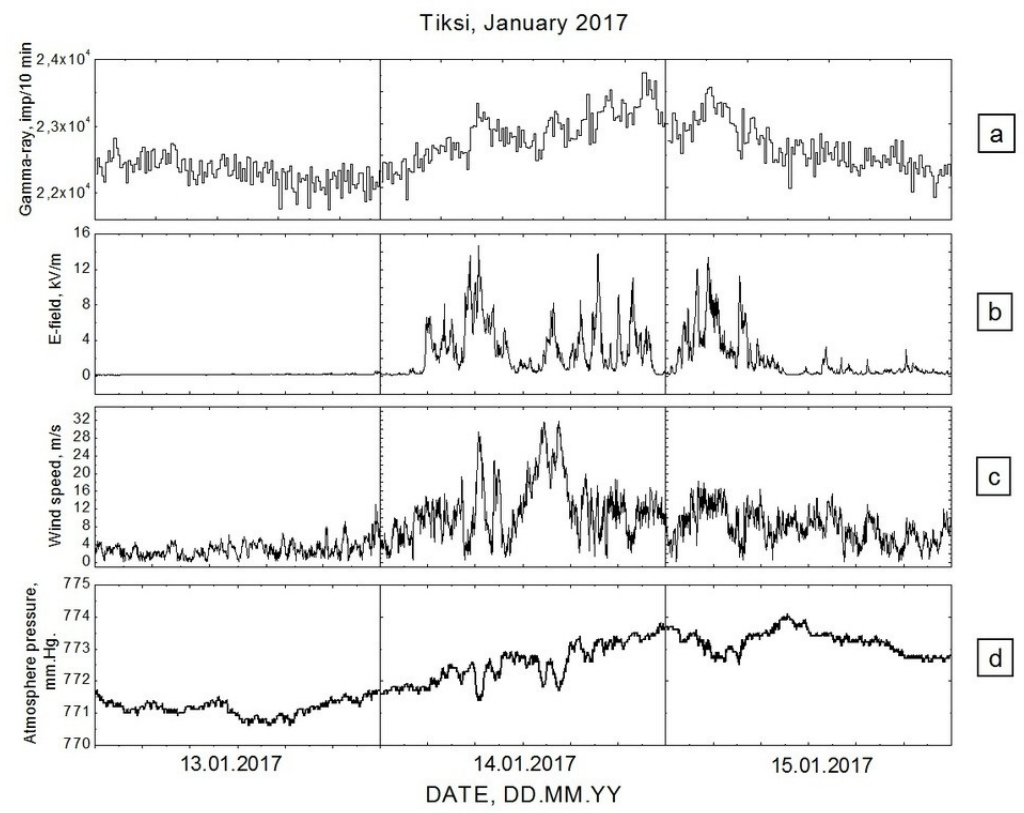

Fig. 4. Strong variations in gamma radiation (a), atmospheric electric field (b), wind speed (c) and atmospheric pressure (d) during a strong snowstorm on January 2017 in Tiksi.

The nature of the variations of the muon component of cosmic rays as well as the intensity of gamma radiation and the intensity of the atmospheric electric field is shown in Figure 5. The monotonous increase in the intensity of the gamma background (a) begins almost synchronously (with a slight delay) with increasing atmospheric pressure (d) and increasing wind speed (c) January 13, 2017. Strong variations in the electric field (b) begin with a delay relative to the increase in gamma radiation 14 January 2017 . The closest connection of gamma radiation is found with atmospheric pressure as in the event in Yakutsk.

The nature of the variations of the muon component of cosmic rays as well as the intensity of gamma radiation and the intensity of the atmospheric electric field is shown in Figure 5.

It is clearly seen that strong changes in the intensity of gamma radiation occur on September 7, 2017 and the most significant muon variations are observed the next day on September 8, 2017. Analysis of the data showed that the strong increase in gamma radiation on September 7, 2017 is primarily due to intense precipitation in the form of storm rain, which also manifests itself in variations of the atmospheric electric field in the form of negative bay-like increases. 


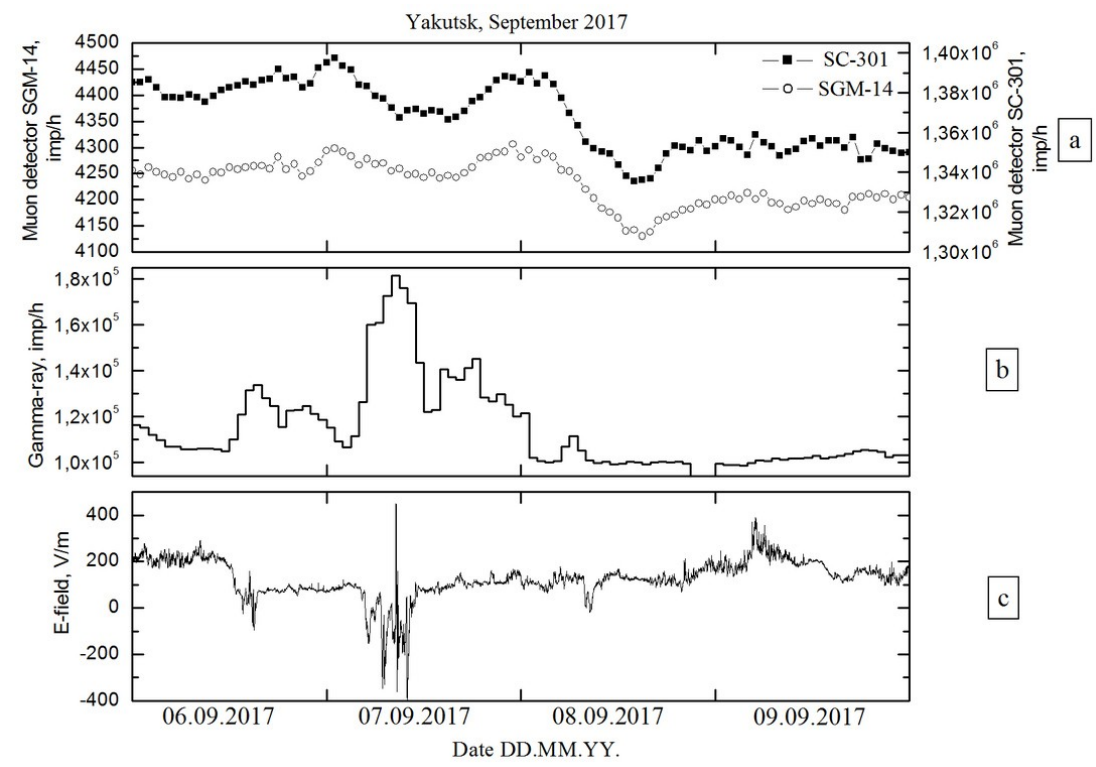

Fig. 5. Variations of the muon component (a), gamma radiation (b), and the atmospheric electric field (c) during heavy precipitation in September 2017 in Yakutsk.

\section{Conclusions}

Analysis of observations in Yakutsk and Tiksi of variations in natural gamma radiation during near thunderstorms, precipitation and blizzards showed that the observed strong variations in gamma radiation are primarily due to the meteorological state of the atmosphere, precipitation and emission of radon and its daughter products from the ground the atmosphere.

It is shown that strong electric fields of a thunderstorm atmosphere are not the main cause of a strong increase in surface gamma radiation.

A close relationship has been found between variations in gamma radiation and wind speed, and, to a lesser extent, with the value of atmospheric pressure.

In the gamma-ray spectra, characteristic lines of the daughter products of the decay of radon $\mathrm{Pb}-214$ and $\mathrm{Bi}-214$ were detected.

The study was supported by RFBR, research projects No. 18-45-140028 r_a.

\section{References}

1. A.V. Germanenko et al. Vestnik Kolskogo nauchnigo centra RAN, 3, 104-109 (2010)

2. A.P.Chubenko, A.L. Shepetov, V.P. Antonova et al., J.Phys. G: Nucl.Part.Phys. 35, 085202, (2008)

3. R. L., Grasty, Geophysics, 62:5, 1379-1385, (1997)

4. U.V. Balabin et al. Geomgnetism and aeronomy, 3, 376-386, (2014)

5. L.P. Babich, Geomag. and Aeron.,47, 664 (2007)

6. D. Koch, M. Mann M, Tellus, 48, 387-396, (1996)

7. S.V. Druzinin et al. Vestnik pomorskogo universiteta. Seriya "Estestvennie nauki", 4, 15-19, (2010) 
8. M. Yamamoto et al. Journal of Environmental Radioactivity, 86, 110-131 (2006)

9. A.A. Toropov A. A. et al. Journal of Atmospheric and Solar-Terrestrial Physics, 94, C. $13-18(2013)$ 